\title{
Feasibility of line-ratio spectroscopy on helium and neon as edge diagnostic tool for Wendelstein 7-X
}

T. Barbui, M. Krychowiak, R. König, O. Schmitz, J. M. Muñoz Burgos, B. Schweer, A. Terra, and

Citation: Review of Scientific Instruments 87, 11E554 (2016); doi: 10.1063/1.4962989

View online: http://dx.doi.org/10.1063/1.4962989

View Table of Contents: http://aip.scitation.org/toc/rsi/87/11

Published by the American Institute of Physics

\section{Articles you may be interested in}

Overview of diagnostic performance and results for the first operation phase in Wendelstein 7-X (invited)

Review of Scientific Instruments 87, 11D304 (2016); 10.1063/1.4964376

Spectroscopic imaging of limiter heat and particle fluxes and the resulting impurity sources during Wendelstein 7-X startup plasmas

Review of Scientific Instruments 87, 11 D606 (2016); 10.1063/1.4959274

Synthetic plasma edge diagnostics for EMC3-EIRENE, highlighted for Wendelstein 7-X

Review of Scientific Instruments 87, 11D441 (2016); 10.1063/1.4959910

Time-dependent analysis of visible helium line-ratios for electron temperature and density diagnostic using synthetic simulations on NSTX-U

Review of Scientific Instruments 87, 11E502 (2016); 10.1063/1.4955286

Hybrid time dependent/independent solution for the $\mathrm{He}$ I line ratio temperature and density diagnostic for a thermal helium beam with applications in the scrape-off layer-edge regions in tokamaks

Physics of Plasmas 19, 012501 (2012); 10.1063/1.3672230

A high resolution IR/visible imaging system for the W7-X limiter

Review of Scientific Instruments 87, 11 D607 (2016); 10.1063/1.4960596

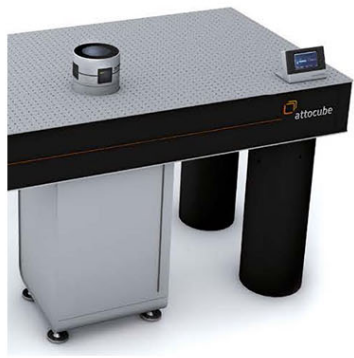

Obstruction free access

optical table with integrated cryocooler

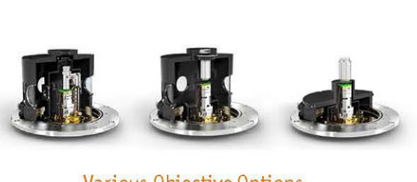

Various Objective 0ptions

\section{attoDRY800}

- Cryogenic Temperatures

- Ultra-Low Vibration

- Optical Table Included

- Fast Cooldown
$5 \%$ DISCOUNT

on all nanopositioners purir
for youra atto Dry80o set up
Coupon Cooce: PJJAD800

attocube 


\title{
Feasibility of line-ratio spectroscopy on helium and neon as edge diagnostic tool for Wendelstein 7-X
}

\author{
T. Barbui, ${ }^{1, a)}$ M. Krychowiak, ${ }^{2}$ R. König, ${ }^{2}$ O. Schmitz, ${ }^{1}$ J. M. Muñoz Burgos,${ }^{3}$ B. Schweer, ${ }^{4}$ \\ A. Terra, ${ }^{5}$ and W7-X Team $\left.{ }^{2, b}\right)$ \\ ${ }^{1}$ Department of Engineering Physics, University of Wisconsin-Madison, Madison, Wisconsin 53706, USA \\ ${ }^{2}$ Max-Planck-Institut für Plasmaphysik, 17491 Greifswald, Germany \\ ${ }^{3}$ Department of Physics and Astronomy, Johns Hopkins University, Baltimore, Maryland 21218, USA \\ ${ }^{4}$ Laboratory for Plasma Physics, Ecole Royale Militaire - Koninklijke Militaire School, 1000 Brussels, Belgium \\ ${ }^{5}$ Forschungszentrum Jülich GmbH, Institut für Energie- und Klimaforschung-Plasmaphysik, \\ Partner of the Trilateral Euregio Cluster (TEC), 52425 Jülich, Germany
}

(Presented 8 June 2016; received 14 June 2016; accepted 6 September 2016; published online 27 September 2016)

\begin{abstract}
A beam emission spectroscopy system on thermal helium $(\mathrm{He})$ and neon $(\mathrm{Ne})$ has been set up at Wendelstein $7-X$ to measure edge electron temperature and density profiles utilizing the line-ratio technique or its extension by the analysis of absolutely calibrated line emissions. The setup for a first systematic test of these techniques of quantitative atomic spectroscopy in the limiter startup phase (OP1.1) is reported together with first measured profiles. This setup and the first results are an important test for developing the technique for the upcoming high density, low temperature island divertor regime. Published by AIP Publishing. [http://dx.doi.org/10.1063/1.4962989]
\end{abstract}

\section{INTRODUCTION}

Line ratio (LR) spectroscopy on helium is widely used in fusion devices as a diagnostic to measure electron temperature and density profiles in the scrape-off-layer and the plasma edge. It is based on the density and temperature dependence of the ratio of selected emission lines of neutral He. In the low temperature $(\ll 10 \mathrm{eV})$ and high density $\left(>10^{20} \mathrm{~m}^{-3}\right)$ conditions expected in the W7-X island divertor, He lines are weak (or not detectable for $T_{e}<5 \mathrm{eV}$ ) and the $T_{e} / n_{e}$ measurement might suffer from great uncertainties. In order to overcome this limitation, LR spectroscopy extended by analysis of absolute line intensities can be performed on $\mathrm{Ne}$ instead of $\mathrm{He}$ or in a $\mathrm{Ne}+\mathrm{He}$ mixture. ${ }^{1} \mathrm{Ne}$ has lower excitation energy and can provide stronger emission lines at low temperatures. The startup campaign of Wendelstein $7-\mathrm{X}$, in which the divertor is not yet installed and five graphite limiters define the plasma boundary, has been used to commission the injection and observation systems with both $\mathrm{He}$ and $\mathrm{Ne}$. We derived initial time and space resolved profiles of electron temperature and density in the scrape-off layer from this diagnostic applying the LR spectroscopy on thermal He. Initial measurements of suitable $\mathrm{Ne}-\mathrm{I}$ lines from pure $\mathrm{Ne}$ and mixed $\mathrm{Ne} / \mathrm{He}$ gas injections were performed as a basis to develop a suited collisionalradiative model $(\mathrm{CRM})$ for $\mathrm{Ne}$ and the combined $\mathrm{He} / \mathrm{Ne}$ situation.

Note: Contributed paper, published as part of the Proceedings of the 21st Topical Conference on High-Temperature Plasma Diagnostics, Madison, Wisconsin, USA, June 2016.

a) Author to whom correspondence should be addressed. Electronic mail: barbui@wisc.edu.

b) Members of the W7-X Team are listed in Nucl. Fusion 53, 126001 (2013).

\section{EXPERIMENTAL SETUP}

The gas injection system provides two in vacuo plugins (one for the upper and one for the lower plasma edge) consisting of gas and water lines as well as a gas box. In the gas box, five piezo valves (opening time of few ms) allow independent gas injection at different poloidal positions. Two valves in the upper injection system are equipped with thin capillary nozzles with inner diameter of $0.6 \mathrm{~mm}$ and length of $1.5 \mathrm{~cm}$ for small beam divergence. The gas boxes are mounted at a position directly behind the divertor plates (to be installed after OP1.1) in two divertor modules which are connected by the same island flux tube in the $\mathrm{m} / \mathrm{n}=5 / 5$ standard magnetic field configuration of W7-X. The vacuum plug-ins are water cooled, capable of steady-state plasma operations. This system allows puffing different gases $\left(\mathrm{He}, \mathrm{Ar}, \mathrm{Ne}\right.$, and $\mathrm{N}_{2}$ during OP1.1 and after OP1.1 to be extended by some explosive gases like $\mathrm{H}_{2}$ or $\mathrm{CH}_{4}$ ) at pressures from few mbar up to 60 bars (mostly interesting for plasma fueling). For the purpose of LR spectroscopy, the pressure is set to tens of mbar: high enough to detect the light from line emissions but low enough not to affect the global and local plasma parameters. This has been confirmed by varying the pressure and monitoring the plasma parameters $\left(\mathrm{T}_{\mathrm{e}}, \mathrm{n}_{\mathrm{e}}\right.$, Bremsstrahlung). At the pressure of $30 \mathrm{mbar}$, the flux of injected atoms is about $1.5 \times 10^{19}$ atoms $/ \mathrm{s}$. During a single discharge, a series of 4-5 75-ms long helium puffs are carried out. The total number of injected atoms is $5 \times 10^{18}$. Assuming that all $\mathrm{He}$ atoms are completely ionized, this leads to an increase in the electron density of $3 \times 10^{17} \mathrm{~m}^{-3}$. This must be related to typical values of the electron density of $2 \times 10^{19} \mathrm{~m}^{-3}$ in the center and of $5 \times 10^{18} \mathrm{~m}^{-3}$ in the outer part of the confined plasma. Accordingly the injected $\mathrm{He}$ atoms can lead at most to an increase in the electron density in the outer confined region of $6 \%$. However experimentally no increase in the edge electron density has been observed. The thermal 


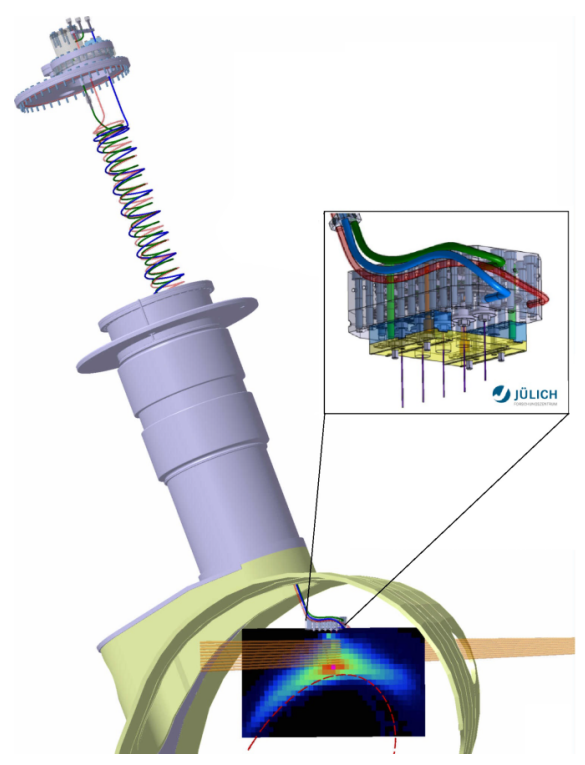

FIG. 1. Overview of the gas injection system at the upper plasma edge. In the zoomed picture, the gas box is shown with all 5 capillary nozzles as planned for the later divertor operation. Also shown are the lines-of-sight (solid orange lines) perpendicular to the beam and the position of the last closed flux surface (red dotted line). The colored area represents a sample EMC3-EIRENE simulation of the He line emission $(\lambda=706.5){ }^{2}$

beam has a mean velocity of $\sim 1.5 \mathrm{~km} \mathrm{~s}^{-1}$ and a divergence of $\sim 40^{\circ}$.

Fig. 1 shows an overview of the injection system at the upper plasma edge along with the lines-of-sight (LOS) of the observation system. The spectroscopic observation is realized here with an array of 8 optical fibers (core diameter $=1 \mathrm{~mm}$, length $=150 \mathrm{~m}$ ) fitted to a camera lens mounted directly behind a vacuum window at a diagnostic observation port of $2 \mathrm{~m}$ length. The LOS are perpendicular to the beam propagation. Their adjustment is based on EMC3-EIRENE simulation of the He line emissions. ${ }^{2}$ This optical setup leads to a spatial resolution of $1 \mathrm{~cm}$ and an observable radial range of $8 \mathrm{~cm}$ in the scrape-off layer. The width of each LOS at the nozzle position is $1 \mathrm{~cm}$. The light is guided to a Czerny-Turner spectrometer, with focal length of $19 \mathrm{~cm}$ and a dispersive grating of 300 grooves $/ \mathrm{mm}$. The spectral resolution is about $0.5 \mathrm{~nm}$. The light is focused at the entrance slit through a lens with objective aperture of $f / 4$. At the spectrometer exit, the light is measured by a CCD camera. The detector is a frame transfer CCD sensor with $512 \times 512$ pixels $(13 \times 13 \mu$ m each $)$. When used in a $512 \times 8$ binning mode, it provides acquisitions with a time resolution of $14 \mathrm{~ms}$. At the gas injection for the lower plasma edge, a single fiber is connected to an echelle spectrometer with a focal length of $25 \mathrm{~cm}$. It provides extended domain spectra $(200-800 \mathrm{~nm})$ with spectral resolution of $50 \mathrm{pm}$ and time resolution of $100 \mathrm{~ms}$. The detector is a frame transfer intensified charge-coupled device (ICCD) with $1024 \times 1024$ pixels $(24 \times 24 \mu \mathrm{m}$ each $)$. This setup is used for first feasibility studies of the beam emission spectroscopy on $\mathrm{Ne}$. For the next divertor campaign starting in mid-2017, the observation systems will be integrated in the 2D scanning spectroscopic endoscopes for divertor plasma observation. ${ }^{3}$

Both observation systems have been absolutely calibrated with an integrating (Ulbricht) sphere. For this, the camera lenses with connected fibers have been dismantled from the diagnostic port and placed in front of the sphere. The spectra acquired while illuminating with the sphere are compared to the spectral radiance provided by the sphere manufacturing company. In this way, a calibration curve is obtained taking into account the transmission of the fibers, the optics, and the grating. This curve is then used to compute the absolute value of the measured line intensities.

\section{EXPERIMENTAL RESULTS}

The measurements presented in this paper were obtained during a standard hydrogen discharge, with electron cyclotron resonance heating (ECRH) injected power of $2 \mathrm{MW}$, central $\mathrm{T}_{\mathrm{e}} \sim 8 \mathrm{keV}$, line-integrated $\mathrm{n}_{\mathrm{e}} \sim 1.5 \times 10^{19} \mathrm{~m}^{-2}$. Repetitive (4-5) 75 ms-long helium puffs were carried out during the discharge through one nozzle in the upper box at reservoir pressure of $\sim 30$ mbar. Spectra were acquired continuously during the discharge with an exposure time of $25 \mathrm{~ms}$. Fig. 2 shows the spectra taken before, during, and after one puff. The measured He I lines are the singlet $\lambda_{1}=667.8$ and $\lambda_{2}=728.1 \mathrm{~nm}$ as well as the triplet $\lambda_{3}=706.5 \mathrm{~nm}$ visible lines. Intrinsic lines are those of C II at 678 and $711 \mathrm{~nm}$, respectively.

Subtracting the background and multiplying by the calibration factor allow obtaining the absolute value of the line intensities. A Gaussian curve fitting is then applied to the lines and its integral gives the line intensity. Fig. 3 shows the intensities of the three He lines for the different lines-ofsight. The innermost LOS is lying $6 \mathrm{~cm}$ outside of the last closed flux surface, while the outermost is in a distance of $3.3 \mathrm{~cm}$ from the nozzle.

From these line intensities, two ratios are computed: $\mathrm{I}_{706} / \mathrm{I}_{728}$ which mainly depends on $\mathrm{T}_{\mathrm{e}}$, and $\mathrm{I}_{668} / \mathrm{I}_{728}$ which is sensitive to $n_{e}$. The derivation of $T_{e}$ and $n_{e}$ is done by comparing these experimental ratios with calculated ratios from a collisional-radiative model (CRM). This model simulates the spectral emission of the neutral He taking into account all significant populating and depopulating mechanisms for the considered atomic levels. The successful application of the line-ratio technique depends strongly on the accuracy of the set of atomic data used in the CRM. In this work, two different models have been applied. The first is a stationary CRM that was previously routinely used at TEXTOR for

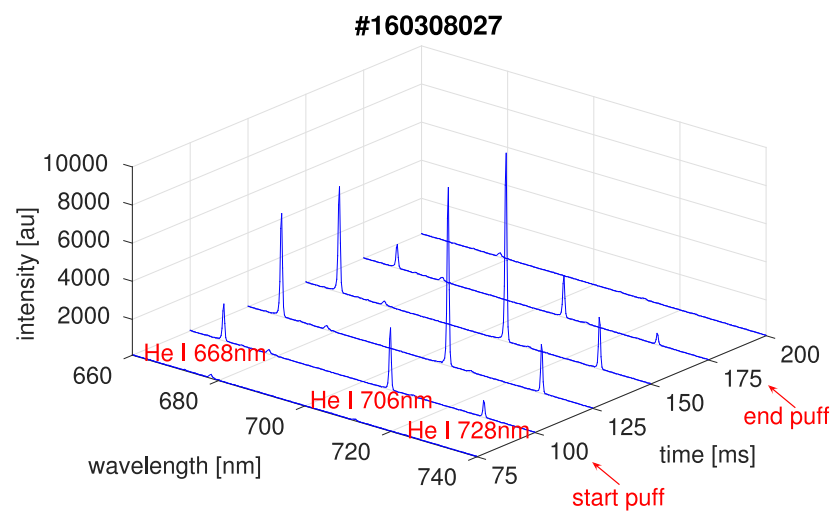

FIG. 2. Spectra acquired before, during, and after a He gas puff. 


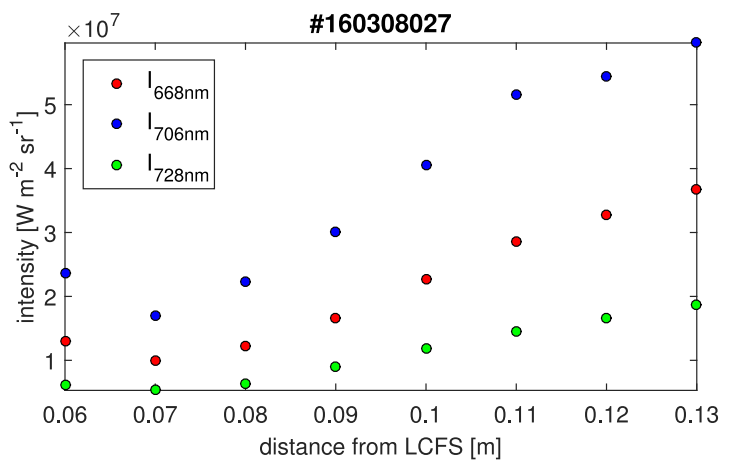

FIG. 3. Radial profiles of absolute line intensities of the three He lines.

the He-beam diagnostic. ${ }^{4}$ The second is a new hybrid time dependent/independent CRM with a renewed set of atomic data. ${ }^{5}$ This model takes into account the long relaxation time of the ${ }^{3} S$ metastable term of helium that populates the triplet spin system. The time dependent solution is important only at lower densities $\left(<1-2 \times 10^{18} \mathrm{~m}^{-3}\right)$ at which the relaxation times for the metastable-triplet system $(\sim 10 \mu \mathrm{s})$ are longer than the time constant of the beam propagation.

Fig. 4 reports the $T_{e}$ and $n_{e}$ profiles determined with the two different CRMs. The profiles are averaged on a single puff. The beam reaches the first LOS after a time greater than the metastable-triplet system relaxation time. Moreover $T_{e}$ and $n_{e}$ do not vary so much in the investigated region. Thus the time dependent solutions give the same results as the equilibrium solutions considering the new hybrid model. The differences with the old TEXTOR model lie in the different atomic database employed in the CRM.
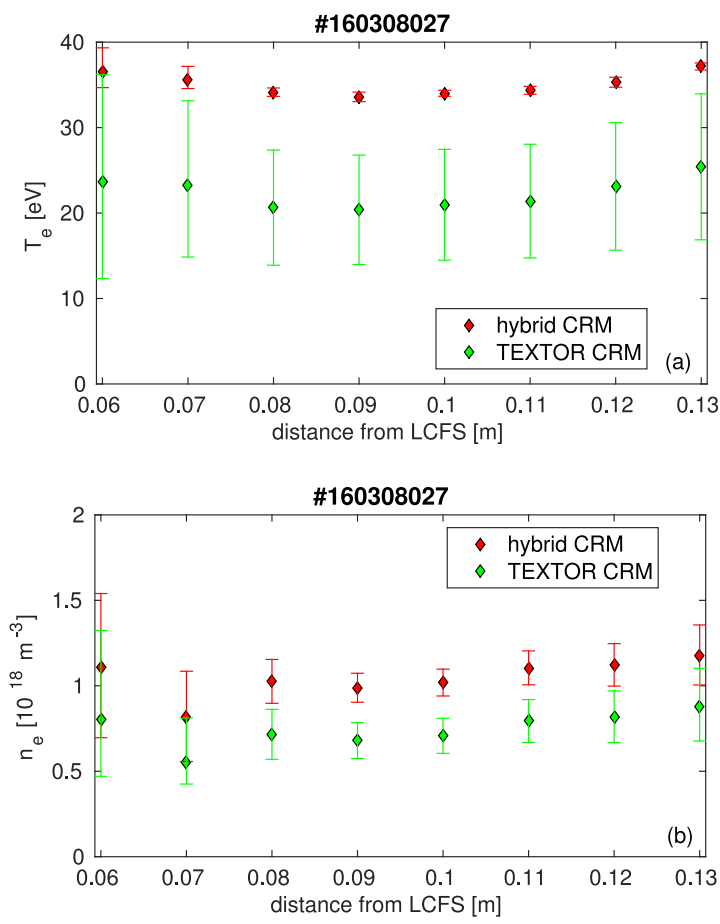

FIG. 4. Edge radial profiles of electron temperature (a) and density (b) from He-beam line-ratio technique.

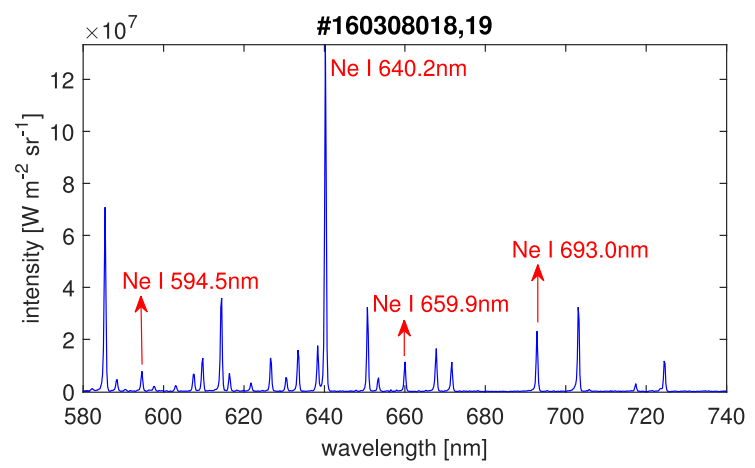

FIG. 5. Ne spectrum with labeled Ne lines suitable for $T_{e} / n_{e}$ determination.

For the TEXTOR CRM, error bars are given by the combination of experimental uncertainties (deviations from the Gaussian fit of the line intensities and time variations over the puff length) and systematic uncertainties. The latter are assumed to be $30 \%$ and $10 \%$ for $\mathrm{T}_{\mathrm{e}}$ and $\mathrm{n}_{\mathrm{e}}$, respectively, as found at TEXTOR by comparing the results of the He beam spectroscopy with other edge diagnostics. ${ }^{4}$ For the hybrid CRM, no comparisons of $n_{e}$ and $T_{e}$ values with other diagnostics have been done so far to validate the set of applied atomic data. We estimate the error bars by propagating the lineratio uncertainties to the derived electron temperatures and densities. These uncertainties are given by the combination of the experimental uncertainties and the CRM uncertainties. The latter are not yet rigorously calculated but roughly estimated to be $2.5 \% .^{5}$ Thus, the error bars of $\mathrm{T}_{e}$ and $\mathrm{n}_{\mathrm{e}}$ for this new CRM have to be considered as preliminary.

The solutions given by the new hybrid model are to be preferred to the ones given by the old TEXTOR model since the hybrid CRM includes a newer and more accurate set of atomic data. It has already been showed in TEXTOR that this new model gives better agreement with other diagnostics than the old model. ${ }^{5}$ Comparisons with other edge diagnostics at $\mathrm{W} 7-\mathrm{X}$ are now being conducted, in particular with the Langmuir probes in the limiter ${ }^{6,7}$ and at the multi-purpose fast manipulator ${ }^{3,7}$ installed during the first operation phase.

First test injections with $\mathrm{Ne}$ have been performed in both gas injection boxes to collect spectra that are useful for the development of a Ne CRM. This model will be used during the next divertor campaign to estimate $\mathrm{T}_{e}$ and $\mathrm{n}_{\mathrm{e}}$ from line emission analysis of neutral $\mathrm{Ne}$. Ne was injected as a single gas (at reservoir pressure of $15 \mathrm{mbar}$ ) and in a mixture with $\mathrm{He}(15$ mbar $\mathrm{Ne}+30$ mbar $\mathrm{He})$. Many $\mathrm{Ne}$ lines have been observed as shown in the spectrum of Fig. 5 for a discharge with Ne puff. Initial line-ratio calculations using the Atomic Data and Analysis Structure (ADAS) ${ }^{8} \mathrm{Ne}$ CRM show that the ratios $640.2 \mathrm{~nm} / 594.5 \mathrm{~nm}$ and $693.0 \mathrm{~nm} / 659.9 \mathrm{~nm}$ are sensitive to $\mathrm{T}_{\mathrm{e}}$ and $\mathrm{n}_{\mathrm{e}}$, respectively.

\section{CONCLUSIONS}

In this paper, the thermal $\mathrm{He} / \mathrm{Ne}$ beam diagnostic for the measurement of $T_{e}$ and $n_{e}$ at Wendelstein 7-X is described and its first results are presented.

The gas injection and spectroscopic observation systems have been successfully commissioned during the first plasma 
operation campaign. Profiles of $T_{e}$ and $n_{e}$ have been derived from the line-ratio technique on the He beam. First injections with $\mathrm{Ne}$ have been carried out and are being used as a test bed for the development of a Ne collisional-radiative model. This model is required for the envisaged determination of $\mathrm{T}_{\mathrm{e}}$ and $\mathrm{n}_{\mathrm{e}}$ through $\mathrm{Ne}$ line emission analysis during the next divertor campaign. The line intensities obtained from $\mathrm{He}$ and $\mathrm{Ne}$ are promising in view of an extended radial coverage due to the lower population energies for the neutral states of $\mathrm{Ne}$ compared to He. Exploiting the aligned emission features for high density, low temperature island divertor measurements seems to be possible.

\section{ACKNOWLEDGMENTS}

This work has been funded in part by the Department of Energy under Grant No. DE-SC0014210. The versatile gas injection system has been developed in collaboration between FZ Jülich, IEK-4, and IPP Greifswald. This work has been carried out within the framework of the EUROfusion Consortium and has received funding from the Euratom research and training programme 2014-2018 under Grant Agreement No. 633053. The views and opinions expressed herein do not necessarily reflect those of the European Commission.

${ }^{1}$ M. Krychowiak, M. Brix, D. Dodt, Y. Feng, R. Koenig, O. Schmitz, J. Svensson, and R. Wolf, Plasma Phys. Controlled Fusion 53, 035019 (2011).

${ }^{2} \mathrm{~F}$. Effenberg et al., "Numerical investigation of plasma edge transport and limiter heat fluxes in Wendelstein 7-X startup plasmas with EMC3-Eirene," Nucl. Fusion (submitted).

${ }^{3}$ O. Neubauer et al., Fusion Eng. Des. 96-97, 891-894 (2015).

${ }^{4}$ O. Schmitz et al., Plasma Phys. Controlled Fusion 50, 115004 (2008).

${ }^{5}$ J. M. Muñoz Burgos, O. Schmitz, S. D. Loch, and C. P. Ballance, Phys. Plasmas 19, 012501 (2012).

${ }^{6} \mathrm{R}$. Koenig et al., J. Instrum. 10, P10002 (2015).

${ }^{7} \mathrm{M}$. Krychowiak et al., "Overview of diagnostic performance and results for the first operation phase in Wendelstein 7-X," Rev. Sci. Instrum. (these proceedings).

${ }^{8}$ See http://www.adas.ac.uk for ADAS-Atomic Data and Analysis Structure. 